\title{
ON COSYMPLECTIC CAUCHY-RIEMANN SUBMANIFOLDS OF LOCALILY CONFORMAL KAEHLER MANIFOLDS
}

\author{
FRANCESCA VERROCA
}

\begin{abstract}
We study some properties of the cosymplectic Cauchy-Riemann submanifolds in a locally conformal Kaehler manifold.
\end{abstract}

\section{Introduction}

The geometry of the Cauchy-Riemann (C.R.) submanifolds of a locally conformal Kaehler. (1.c.K.) manifold has been studied in the last ten years, ([4], [5], [6], [10], [12], [13], [18], [19]).

The concept of normal C.R. submanifold was introduced by A. Bejancu ([1]) in analogy with the theory of the normal almost contact structures, ([3], [7]).

In [1] a theory for the normal C.R. submanifolds in a Kaehler manifold is developed. In particular, a C.R. hypersurface of a Kaehler manifold is a normal contact hypersurface, ([14]).

Some properties of the normal C.R. submanifolds of 1.c.K. manifolds have been studied in former papers, ([18], [19]).

In this paper, we study the cosymplectic C.R. submanifolds in a l.c.K. manifold.

\section{Preliminaries}

Let $\left(M^{2 n}, g_{0}, J\right)$ be a Hermitian manifold of complex dimension $n$, with Kaehler 2-form $\Omega_{0}$, i.e. $\Omega_{0}(X, Y)=g_{0}(X, J Y), X, Y \in T M^{2 n}$.

Then $\left(M^{2 n}, g_{0}, J\right)$ is a locally conformal Kaehler (l.c.K.) manifold if there exists a closed 1-form $\omega_{0}$ on $M^{2 n}$ such that

$$
d \Omega_{0}=\omega_{0} \wedge \Omega_{0}
$$

Received April 20, 1993.

1991 Mathematics Subject Classification. 53C40, 53C35

Key words and phrases. Cauchy-Riemann submanifolds.

Work partially supported by MURST. 
The 1 -form $\omega_{0}$ is called the Lee form, the Lee vector field is the vector field $B_{0}$ such that $g_{0}\left(B_{0}, X\right)=\omega_{0}(X), X \in T M^{2 n}$.

If $\bar{\nabla}$ denotes the Riemannian connection of $\left(M^{2 n}, g_{0}\right)$, then one has:

$$
\left(\bar{\nabla}_{X} J\right) Y=\frac{1}{2}\left\{\theta_{0}(Y) X-\omega_{0}(Y) J X-\Omega_{0}(X, Y) B_{0}-g(X, Y) A_{0}\right\} \quad X, Y \in T M^{2 n}
$$

where $\theta_{0}=\omega_{0} \circ J$ is the anti-Lee 1 -form and $A_{0}=-J B_{0}$ is the anti-Lee vector field. We use the notation and the properties stated in [16], [17].

A submanifold $M^{m}$ of $M^{2 n}$ is called a Cauchy-Riemann (C.R.) submanifold of $M^{2 n}$ if the tangent bundle $T M^{m}$ is expresed as a direct sum of two distributions $O$ and $O^{\perp}$, such that $O$ is holomorphic (i.e. $J_{x}\left(O_{x}\right)=O_{x}, x \in M^{m}$ ) and $O^{\perp}$ is totally real (i.e. $\left.J_{x}\left(O_{x}^{\perp}\right) \subset\left(T_{x} M^{m}\right)^{\perp}, x \in M^{m}\right)$.

Let $p$ be the complex dimension of the holomorphic distribution $O$ and let $q$ be the real dimension of the totally real distribution $O^{\perp}$.

If $q=0, M^{m}$ is called holomorphic submanifold; if $p=0, M^{m}$ is called totally real submanifold.

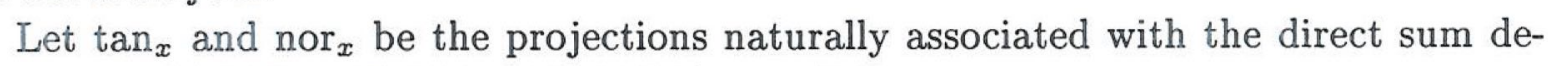
composition $T_{x} M^{2 n}=T_{x} M^{m} \oplus\left(T_{x} M^{m}\right)^{\perp}, x \in M^{m}$.

We put $P X=\tan (J X), F X=\operatorname{nor}(J X), t \xi=\tan (J \xi)$ and $f \xi=\operatorname{nor}(J \xi)$ for any $X \in T M^{m}, \xi \in\left(T M^{m}\right)^{\perp}$.

Then, for any $X \in T M^{m}$ one has $P X \in O$.

Moreover, the following identities hold: $P^{2}=-I-t F, f^{2}=-I-F t, F P=0, f F=$ $0, t F=0, P t=0, P^{3}+P=0, f^{3}+f=0,([11])$.

The Gauss and Weingarten formulas are still valid, that is:

$$
\bar{\nabla}_{X} Y=\nabla_{X} Y+h(X, Y), \quad \bar{\nabla}_{X} \xi=-A_{\xi} X+\nabla_{X}^{\perp} \xi
$$

for any $X, Y \in T M^{m}, \xi \in\left(T M^{m}\right)^{\perp}$.

Here $\nabla, h, A_{\xi}$ and $\nabla^{\perp}$ stand, respectively, for the induced connection, the second foundamental form, the Weingarten operator (associated with $\xi \in\left(T M^{m}\right)^{\perp}$ ) and the normal connection in $\left(T M^{m}\right)^{\perp}$.

The forms $\theta, \omega$ and $\Omega$ are naturally induced on the submanifold $M^{m}$ by $\theta_{0}, \omega_{0}$ and $\Omega_{0}$ respectively. One has:

$$
\theta=\omega \circ P+\omega_{0} \circ F, \quad \Omega(X, Y)=g(X, P Y), \quad X, Y \in T M^{m}
$$

As a consequence of (1.2) and (1.3) one has:

$$
\begin{gathered}
\left(\nabla_{X} P\right) Y=A_{F Y} X+\operatorname{th}(X, Y)+\frac{1}{2}\{\theta(X) Y-\omega(Y) P X-\Omega(X, Y) B-g(X, Y) A\} \\
\left(\nabla_{X} F\right) Y=f h(X, Y)-h(X, P Y)-\frac{1}{2}\left\{\omega(Y) F X+\Omega(X, Y) B^{\perp}+g(X, Y) A^{\perp}\right\}
\end{gathered}
$$


for any $X, Y \in T M^{m}$, where $A=\tan \left(A_{0}\right), B=\tan \left(B_{0}\right), A^{\perp}=\operatorname{nor}\left(A_{0}\right)$ and $B^{\perp}=$ $\operatorname{nor}\left(B_{0}\right)$. We put:

$$
S(X, Y)=[P, P](X, Y)-2 t(d F)(X, Y), X, Y \in T M^{m}
$$

Here $[P, P]$ is the Nijenhuis torsion of $P$ and $d F$ is the differential of the vector valued 1 -form $F$, which can be expressed as follows:

$$
2(d F)(X, Y)=\nabla_{X}^{\perp}(F Y)-\nabla_{Y}^{\perp}(F X)-F[X, Y], \quad X, Y \in T M^{m} .
$$

A C.R. submanifold is called normal if $S=0$, ([1]).

A C.R. submanifold is called cosymplectic if it is normal and $F$ and $\Omega$ are closed. $P$ is called parallel if $\nabla P=0 . F$ is called parullel if $\nabla F=0$.

\section{Cosymplectic C.R. submanifolds}

Lemma 2.1. Let $M^{m}$ be a C.R. submanifold of the l.c.K. manifold $M^{2 n}$. Then, one has:

$$
\begin{aligned}
2 g\left(\left(\nabla_{X} P\right) Y, Z\right)= & 3(d \Omega)(X, P Y, P Z)-3(d \Omega)(X, Y, Z)+g([P, P](Y, Z), P X) \\
& +2 g_{0}((d F)(P Y, Z), F X)+2 g_{0}((d F)(P Y, X), F Z) \\
& -2 g_{0}((d F)(P Z, X), F Y)-2 g_{0}((d F)(P Z, Y), F X)
\end{aligned}
$$

for any $X, Y, Z \in T M^{m}$.

By a easy calculation one has:

$$
\begin{gathered}
2(d F)(P Y, Z)=\nabla \frac{1}{P Y}(F Z)-F[P Y, Z], \quad Y, Z \in T M^{m} \\
3(d \Omega)(X, Y, Z)=X(g(Y, P Z))+Y(g(Z, P X))+Z(g(X, P Y))-g([X, Y], P Z) \\
-g([Z, X], P Y)-g([Y, Z], P X), \quad X, Y, Z \in T M^{m} .
\end{gathered}
$$

Then, for any $X, Y, Z \in T M^{m}$ it follows that:

$$
\begin{aligned}
& 3(d \Omega)(X, P Y, P Z)-3(d \Omega)(X, Y, Z)+g([P, P](Y, Z), P X)+2 g_{0}((d F)(P Y, Z), F X) \\
& +2 g_{0}((d F)(P Y, X), F Z)-2 g_{0}((d F)(P Z, X), F Y)-2 g_{0}((d F)(P Z, Y), F X) \\
= & X(g(P Y,-Z-t F Z))+(P Y)\left(g(Z, X)-g_{0}(F Z, F X)\right)-(P Z)(g(P X, P Y)) \\
& +g(P[X, P Y], P Z)+g(P[P Z, X], P Y)-g([P Y, P Z], P X)+X(g(P Y, Z)) \\
& -Y(g(Z, P X))-Z(g(X, P Y))+g([X, Y], P Z)+g([Z, X], P Y)+g([Y, Z], P X) \\
& +g([P Y, P Z], P X)-g(P[P Y, Z], P X)-g(P[Y, P Z], P X)+g\left(P^{2}[Y, Z], P X\right) \\
& +2 g_{0}((d F)(P Y, Z), F X)+2 g_{0}((d F)(P Y, X), F Z)-g_{0}\left(\nabla \frac{\perp}{P Z}(F X), F Y\right)
\end{aligned}
$$




$$
\begin{aligned}
& +g_{0}(F[P Z, X], F Y)-g_{0}\left(\nabla \frac{\perp}{P Z}(F Y), F X\right)+g_{0}(F[P Z, Y], F X) \\
= & (P Y)(g(Z, X))-(P Y)\left(g_{0}(F Z, F X)\right)-(P Z)(g(X, Y))+g(P[X, P Y], P Z) \\
& +g([P Z, X], Y)-Y(g(Z, P X))-Z(g(X, P Y))+g([X, Y], P Z) \\
& +g([Z, X], P Y)-g([P Y, Z], X)+g_{0}(F[P Y, Z], F X)-g([Y, P Z], X) \\
& +2 g_{0}((d F)(P Y, Z), F X)+2 g_{0}((d F)(P Y, X), F Z) \\
= & X(g(P Y, Z))+(P Y)(g(Z, X))-Z(g(X, P Y))+g([X, P Y], Z) \\
& +g([Z, X], P Y)-g([P Y, Z], X)+X(g(Y, P Z))+Y(g(P Z, X)) \\
& -(P Z)(g(X, Y))+g([X, Y], P Z)+g([P Z, X], Y)-g([Y, P Z], X) \\
= & 2 g((\nabla X P) Y, Z) .
\end{aligned}
$$

Theorem 2.1. Let $M^{m}$ be a C.R. submanifold of th l.c.K. manifold $M^{2 n}$. If $M^{m}$ is cosymplectic then $P$ is parallel.

The statement is a consequence of the lemma 2.1.

Theorem 2.2. Let $M^{m}$ be a C.R. submanifold of the l.c.K. manifold $M^{2 n}$. If $M^{m}$ is cosymplectic then $M^{m}$ is a C.R. product.

The statement is a consequence of the theorem 2.1 and of the theorem 5.1 in [12].

Remark 2.1. Let $M^{m}$ be a C.R. submanifold of the l.c.K. manifold $M^{2 n}$. If $d F=0$ and $M^{m}$ is normal, by (1.7), one has: $[P, P]=0$.

Proposition 2.1. Let $M^{m}$ be a C.R. submanifold of a l.c.K. manifold $M^{2 n}$. Then one has:

$S(X, Y)=\left(\nabla_{P X} P\right) Y-\left(\nabla_{P Y} P\right) X+P\left(\left(\nabla_{Y} P\right) X-\left(\nabla_{X} P\right) Y\right)-J\left(\left(\nabla_{X} F\right) Y-\left(\nabla_{Y} F\right) X\right)$ for any $X, Y \in T M^{m}$.

For any $X, Y, \in T M^{m}$, one has:

$$
\begin{aligned}
& \left(\nabla_{P X} P\right) Y-\left(\nabla_{P Y} P\right) X+P\left(\left(\nabla_{Y} P\right) X-\left(\nabla_{X} P\right) Y\right)-J\left(\left(\nabla_{X} F\right) Y-\left(\nabla_{Y} F\right) X\right) \\
= & {[P X, P Y]-P\left(\nabla_{P X} Y-\nabla_{Y} P X\right)-P\left(\nabla_{X}(P Y)-\nabla_{P Y} X\right)+P^{2}\left(\nabla_{X} Y-\nabla_{Y} X\right) } \\
& -J\left(\nabla \frac{1}{X}(F Y)-\nabla_{Y}(F X)-F\left(\nabla_{X} Y-\nabla_{Y} X\right)\right) \\
= & S(X, Y) .
\end{aligned}
$$

Corollary 2.1. Let $M^{m}$ be a C.R. submanifold of the l.c.K. manifold $M^{2 n}$. If $P$ and $F$ are parallel then $M^{m}$ is normal.

Remark 2.2. Let $M^{m}$ be a C.R. submanifold of the l.c.K. manifold $M^{2 n}$. If $[P, P]=0$, then the distribution $O$ is integrable.

If the distribution $O$ is integrable then $[P, P](X, Y) \in O$ for any $X, Y \in T M^{m}$.

Corollary 2.2. Let $M^{m}$ be a C.R. submanifold of the l.c.K. manifold $M^{2 n}$. If $P$ and $F$ are parallel then the distribution $O$ is integrable. 
Theorem 2.2. Let $M^{m}$ be a C.R. submanifold of the l.c.K. manifold $M^{2 n}$. If $P$ and $F$ are parallel then $M^{m}$ is cosymplectic.

The corollary 2.1 implies that $M^{m}$ is normal.

Since $F$ is parallel it follows that $d F=0$, and the remark 2.1 gives $[P, P]=0$. By means of the lemma 2.1 one has:

$$
(d \Omega)(X, Y, Z)=(d \Omega)(X, P Y, P Z), \quad X, Y, Z \in T M^{m} .
$$

Putting $X=Y$, one has:

$$
(d \Omega)(X, P X, P Z)=0, \quad X, Z \in T M^{m} .
$$

Replacing $X+Y$ with $X$ in (2.3), one has:

$$
\begin{gathered}
(d \Omega)(X, P Y, P Z)=-(d \Omega)(Y, P X, P Z), \quad X, Y, Z \in T M^{m} ; \\
(d \Omega)(X, Y, Z)=0, X, Z \in T M^{m}, \quad Y \in O^{\perp} .
\end{gathered}
$$

Applying (2.2) and (2.5) for any $X, Y, Z \in T M^{m}$ one has:

$$
\begin{aligned}
(d \Omega)(X, Y, Z) & =(d \Omega)(X, P Y, P Z)=-(d \Omega)(Y, P X, P Z)=-(d \Omega)(P X, P Z, Y) \\
& =-(d \Omega)\left(P X, P^{2} Z, P Y\right)=(d \Omega)(P X, Z, P Y)=-(d \Omega)(Z, P X, P Y) \\
& =-(d \Omega)(X, Y, Z) .
\end{aligned}
$$

Therefore $\Omega$ is closed.

Propositon 2.2. Let $M^{m}$ be a C.R. submanifold of the l.c.K. manifold $M^{2 n}$. If $M^{m}$ is generic, $q>1$, normal and $P$ is parallel then $F$ is parallel.

Since $P$ is parallel it follows that $\nabla_{X} Y \in O$ for any $X \in T M^{m}, Y \in O$. Therefore one has $\left(\nabla_{X} F\right) Y=0$ for any $X \in T M^{m}, Y \in O$.

By (1.7) one has:

$$
(\nabla X F) Y=f h(X, P Y)-\frac{1}{2}\left\{\omega(Y) F X+\Omega(X, Y) B^{\perp}+g(X, Y) A^{\perp}\right\}, X, Y \in T M^{m} .
$$

Applying the theorem 2.1 in [6] one obtains $\omega=0$ on the distribution $O^{\perp}$ and $A^{\perp}=0$.

Since $M^{m}$ is generic, it follows that $f=0$.

Therefore one has $\left(\nabla_{X} F\right) Y=0$ for any $X \in T M^{m}, Y \in O^{\perp}$.

Corollary 2.3. Let $M^{m}$ be a generic C.R. submanifold, $q>1$, of the l.c.K. manifold $M^{2 n}$. The following statements are equivalent:

a) $M^{m}$ is cosymplectic;

b) $P$ and $F$ are parallel. 


\section{References}

[1] A. Bejancu, "Normal C.R. submanifolds of Kaehler manifolds," Ann. Univ. "A1 I Cuza", Iasi, 26, (1980), 123-132.

[2] A. Bejancu, Geometry of C.R. submanifolds, D. Reidel Publ. Co, Dordrecht, 1986.

[3] D.E. Blair, Contact manifold in Riemannian geometry, Lecture notes in Math., 509, SpringerVerlag, Berlin, 1976.

[4] D.E. Blair and B.Y. Chen, "On C.R. submanifolds of Hermitian manifolds," Israel J. Math., 34, (1979), 353-363.

[5] S. Dragomir, "Cauchy-Riemann submanifolds of locally conformal Kaehler manifolds II," Atti Sem. Mat. Fis. Univ. Modena, 37, (1989), 1-11.

[6] S. Dragomir and F. Verroca, Normal Cauchy-Riemann submanifolds of locally conformal Kaehler manifolds, Rapporto interno 90/1 Dip. Mat. Univ. Bari.

[7] Y. Hatakeyama and S. Sasaki, "On differentiable manifolds with contact metric structures," $J$. Math. Soc. Japan, 14, (1962), 249-271.

[8] S. Ianus, "Sulla struttura fogliata di una varietà cosimplettica," Bull. Math. Soc. Sci. Math. Romania, Toml 20, (68), (1976), 1-2.

[9] S. Ianus, K. Matsumoto and L. Ornea, "Immersion spheriques dans une varieté de Hopf gèneralisèe," C.R. Acad. Paris, t. 316, serie I, (1966), 63-66.

[10] S. Ianus and L. Ornea, "A class of anti-invariant submanifolds of a generalized Hopf manifolds," Bull. math. Soc. Sci. Math. Romania, 34, (1990), 115-123.

[11] M. Kon and K. Yano, "C.R. submanifolds of Kaehlerian and Sasakian manifolds," Progress in Math., Vol. 30, Ed. By J. Coates and S. Helgason, Birhhauser, Boston-Basel-Stuttgart, 1983.

[12] K. Matsumoto, "On C.R. submanifolds of locally conformal Kaehler manifolds," Journal of the Korean. Math. Soc., vol.21, n.1, (1984), 49-61.

[13] K. Matsumoto, "On C.R. submanifolds of locally conformal Kaehler manifolds II," Tensor (New Series), Vol. 45, (1987), 144-150.

[14] M. Okumura, "Certain almost contact hypersurfaces in Kaehlerian manifold of constant holomorphic sectional curvature," Tokohu Math. J. 16, (1964), 270-284.

[15] L. Ornea, "On C.R. submanifolds of locally conformal Kaehler manifolds," Demonstratio Math., Warsawa, (14), 39, (1986), 863-869. [16] I. Vaisman, "On locally conformal almost Kaehler manifolds," Israel J. of Math., 24, (1976), 338-
351. [17] I. Vaisman, "Locally conformal Kaehler manifolds with parallel Lee form," Rendiconti di Mat.,
Roma, 12, (1979), 263-284.

[18] F. Verroca, "On a class of Cauchy-Riemann submanifolds of locally conformal Kaehler manifolds," Bull. Math. Soc. Sci. Math. Romania, 4, (1991), 89-97.

[19] F. Verroca, "On Sasakian anti-holomorphic Cauchy-Riemann submanifolds of locally conformal Kaehler manifolds," Publicationes Mathematicae (Debrecen), Vol. 42, (1993).

Università degli Studi di Bari, Dipartimento di Matematica, Campus Universitario, Via Edoardo Orabona 4, 70125 Bari Italy. 